\title{
EDITORIAL
}

\section{Simulation training for emergency medicine residents: time to move forward}

\author{
Trevor S. Langhan, MD
}

VERSION FRANÇAISE À LA PAGE 470

\section{Introduction}

Unstable patients requiring a critical procedure may suffer unnecessary morbidity because of delays incurred or from a lack of technical ability while a resident is learning a new procedure. If teaching does not occur at that time, when can a resident expect to acquire these skills? Historically, residents have learned their skills in the opportunistic, unstructured environment of the emergency department. The mantra "see one, do one, teach one" is often repeated to residents throughout their training. Although senior staff provide supervision, inevitably, errors that otherwise would not occur are made as residents learn their skills.

Procedural skill task trainers and simulation-based modalities are gaining acceptance in the medical education community. For the learning of critical interventions, simulation-based training is structured so that the acquisition of new skills does not harm patients. Unfortunately, while there are many strengths to simulation-based education, the direction and growth of this field has, at times, been controlled by interests that are not necessarily focused on the patient or the education process.

\section{A brief history}

Emergency medicine (EM) is unpredictable and diverse. A retrospective study by Hayden and Panacek examined the procedural skill experience of a heterogeneous group of first-year residents. In their analysis, the authors found significant variability in the types and numbers of procedures performed by residents during their undergraduate education. ${ }^{1}$ A second study found that, for EM residents, the number of patients seen per shift increased with increasing seniority, as did the variability of procedures accomplished. Given the large variability in procedural skill proficiency witnessed in junior residents, ${ }^{2}$ the challenge is to ensure a uniformity of experience for all trainees.

Psychomotor skill acquisition is a complex phenomenon. When residents learn the steps of a procedure and practise the skill through repetition, experiential learning dominates. In the clinical context, competing interests for residents' attention hamper this type of task breakdown thus limiting acquisition of the skill. The ability to focus the learning directly on the task at hand and eliminate all distractions is one of the hallmarks of simulation. ${ }^{3-6}$

Simulation-based education also has a strong foundation in experiential learning theory. In the experiential learning cycle, the learner is exposed to concrete experiences and is provided the opportunity for reflection and abstraction. Simulation-based medical education offers a traineecentred environment that allows the educator to provide full attention to an individual learner's needs at an appropriate pace. ${ }^{7-9}$ The learning cycle of experience and feedback can be repeated in a simulated environment without fear of repercussion. The simulation cycle of "doing," and "reflection/feedback" is the crux of the simulation education process. It is the directed feedback at opportune times during simulation training that have led some authors to cite Lev Vygotsky as an important theorist in simulation training. ${ }^{4}$ Vygotsky's conceptualization of the "zone of proximal development" lends itself well to procedural skill learning. This zone is the theoretical moment where learning takes place, and where intervention by an instructor would have the greatest potential for retention. ${ }^{4}$ Instruction at that time is thought to "awaken" an entire set of skills that are in the stage of maturation. This theory has been adapted by modern

Attending physician, Foothills Medical Centre, University of Calgary, Calgary, Alta.; at the time of submission: Emergency Medicine Resident, University of Calgary, Calgary, Alta.

All editorial matter represents the views of the authors and not necessarily those of CJEM or the Canadian Association of Emergency Physicians.

CJEM 2008;10(5):467-9 
educators into "scaffolding," or the provision of mentorship in a graded fashion. The preceptor offers less guidance as the learner develops more mastery of the skill. ${ }^{6}$

Simulation as a teaching modality has been a part of medical education for centuries. Beginning with cadaver study in the 15th century, through the use of the Resusci Anne (Laerdal Medical) and Harvey (Center for Research in Medical Education, University of Miami) cardiology manikins in the 1960s and 1980s, respectively, and continuing with the Gainesville Anesthesia Simulator (Medical Education Technologies, Inc.), the evolution of simulation has been intimately entwined with the evolution of technology. Although more commonly thought of as a computerdriven interactive model, true simulation includes cadavers, anatomy sections, anatomical models and homemade task trainers. It also comprises such complex instruments as virtual reality and interactive models with tactile responses. ${ }^{3,410-13}$ The most common operating definition of simulation in medical education literature is "any educational activity that uses aids to enhance the medical educational message." ${ }^{5}$

\section{The ethics of simulation}

Four major themes have evolved with regard to the ethics of simulation. ${ }^{6}$ The first is an assurance of best standards. This ethical drive for best standards pertains to education and assessment. In education, simulation allows the learner to practise skills in a learner-centred environment and ensures mastery of skills before accessing patients. The "best standards" in relation to evaluation argues that simulation can be used to develop a measurement tool that is valid, is not prone to measurement errors and is standardized. This tool could then be used to eliminate bias from clinical and procedural skill exam stations. . $6,11,14-22^{-1,16}$

The second ethical theme supporting simulation training is error management. The most important theoretical advantage of simulation and the one most pertinent to an ethical discussion is the ability to allow learners to make mistakes and to allow errors to progress in their entirety. ${ }^{6}$ With real patients, all errors must be stopped as soon as they are recognized, depriving students of potential learning in the interest of patient safety. Simulation allows the evolution of errors without fear of patient harm. Advocacy for patient rights and safety has become one of the major driving forces for simulation in medical education. ${ }^{3}$ A leading expert in medical education stated "no industry in which human lives depend on skilled performance has waited for unequivocal proof of the benefits of simulation before embracing it." 6

The third ethical theme in simulation-based medical education is patient autonomy: an effort to recognize the right of patients to direct their own care. ${ }^{6}$ Some patients refuse to let learners be involved in their care, and this loss of a "learning resource" can be made up with simulation.

The fourth and most subtle of the ethical themes is social justice. This theme focuses on the fact that the burden of educating physicians has historically been disproportionately carried out by the lower socioeconomic classes. Teaching hospitals often service a larger proportion of the urban poor than peripheral community hospitals. Some have argued that with simulation a more appropriate balance can be achieved to spread the burden of educating the next generation of physicians. ${ }^{3,6}$

Despite the growing acceptance of simulation-based medical education, it is difficult to find empirical evidence supporting it. There are a limited number of quantitative studies that have demonstrated a change in behaviour because of simulation training. In a systematic review of the literature, Ravert ${ }^{23}$ searched for quantitative studies in the field of simulation. Only 9 of 513 studies met inclusion criteria for their analysis. Their review demonstrated the paucity of empirical evidence to support the broad implementation of simulation in medical education. ${ }^{2428}$ To date there are no conclusive evidence-based studies that prove the superiority of simulation-based medical education over traditional teaching methodology.

A number of potential pitfalls exist in the use of simulation in training. The knowledge translation from the simulated environment to the clinical world has not been fully explored. Other limitations for the use of simulation include, but are not limited to, the need to overcome resistance to change, the creation of a constructive atmosphere for learning, the training of the current educators in delivery of simulation-based education, the evolution of the valid assessment tool, the high start-up and technical support costs, and the development of a sustainable business model. ${ }^{3,5,6}$

\section{Influence of industry}

Before the medical education community embraces more technologically advanced (and hence more expensive) simulators, the role of lower-cost, less technologically advanced simulators must be evaluated. The machine that drives medical education should not be the same machine that drives the evolution of technology. Said another way, the makers of simulators may have different goals than the users of the technology. ${ }^{4}$ Most importantly, we must not allow technology to drive the educational agenda, but instead develop technology that meets our needs. ${ }^{13}$ It is our responsibility as educators to prevent the natural creep of for- 
profit business guiding medical education pedagogy. While theoretical and ethical reasons for pursuing simulation training exist, this does not mean higher fidelity equals better learning. There are no empirical prospective studies that support the use of more highly advanced modalities over lower-fidelity models. Simulation-based instruction has become the newest trend in medical education. Ultra high fidelity task trainers and virtual reality computer-based programs, while impressive in their appearance, have not been demonstrated to be superior to low- or moderate-fidelity trainers. A Styrofoam cup and angiocath may replicate the psychomotor construct of starting an intravenous line just as well as a much more expensive polyethylene-based vascular access simulator.

\section{Conclusion}

The diversity of preceptors, varying learner experience, opportunistic procedural skill performance and haphazard evaluation confounds proper learning and assessment during EM rotations. ${ }^{3-6}$ EM residents may have any number of preceptors over the course of their training, limiting longitudinal observation and the evaluation of procedural skill competence. The use of simulation could supplement clinical learning in the emergency department allowing for the consolidation of skills. A further advantage of simulation would be the development of standardized evaluation tools to ensure competence in clinical skills before "real" patient contact.

The classic mantra of "see one, do one, teach one" has fallen out of favour, and learning by doing is no longer acceptable. At a meeting of the Education Technology Section in 2004, a consensus statement pertaining to EM education was formalized. In closing, their conclusion echoes the principle point of this paper: "See one, simulate many, do one competently and teach everyone."

\section{Competing interests: None declared.}

Keywords: simulation, medical education, procedural skills, ethics

\section{References}

1. Hayden SR, Panacek EA. Procedural competency in emergency medicine: the current range of resident experience. Acad Emerg Med 1999;6:728-35.

2. Deveau JP, Lorenz JE, Hughes MJ. Emergency medicine resident work productivity and procedural accomplishment. J Am Osteopath Assoc 2003;103:291-6.

3. Gaba DM. The future vision of simulation in health care. Qual Saf Health Care 2004;13(Suppl 1):i2-10.

4. Kneebone RL, Scott W, Darzi A, et al. Simulation and clinical practice: strengthening the relationship. Med Educ 2004;38: 1095-102.
5. Ziv A, Ben-David S, Ziv M. Simulation based medical education: an opportunity to learn from errors. Med Teach 2005;27: 193-9.

6. Ziv A, Wolpe PR, Small SD, et al. Simulation-based medical education: an ethical imperative. Acad Med 2003;78:783-8.

7. Seropian MA, Brown K, Gavilanes JS, et al. Simulation: not just a manikin. J Nurs Educ 2004;43:164-9.

8. Richardson BK. Feedback. Acad Emerg Med 2004;11:e1-5.

9. Schuwirth LW, van der Vleuten CP. The use of clinical simulations in assessment. Med Educ 2003;37(Suppl 1):65-71.

10. Dawson S. Procedural simulation: a primer. Radiology 2006; 241:17-25.

11. Henriksen K, Moss F. From the runway to the airway and beyond. Qual Saf Health Care 2004;13(Suppl 1):i1.

12. Nestel D, Kneebone R, Black S. Simulated patients and the development of procedural and operative skills. Med Teach 2006; 28:390-1.

13. Vozenilek J, Huff JS, Reznek M, et al. See one, do one, teach one: advanced technology in medical education. Acad Emerg Med 2004;11:1149-54.

14. Beaubien JM, Baker DP. The use of simulation for training teamwork skills in health care: how low can you go? Qual Saf Health Care 2004;13(Suppl 1):i51-6.

15. Dawson S. Procedural simulation: a primer. J Vasc Interv Radiol 2006;17:205-13.

16. Graber MA, Wyatt C, Kasparek L, et al. Does simulator training for medical students change patient opinions and attitudes toward medical student procedures in the emergency department? Acad Emerg Med 2005;12:635-9.

17. Kovacs G. Procedural skills in medicine: linking theory to practice. J Emerg Med 1997;15:387-91.

18. Rosenthal ME, Adachi M, Ribaudo V, et al. Achieving housestaff competence in emergency airway management using scenario based simulation training: comparison of attending vs housestaff trainers. Chest 2006;129:1453-8.

19. Hesselfeldt R, Kristensen MS, Rasmussen LS. Evaluation of the airway of the SimMan full-scale patient simulator. Acta Anaesthesiol Scand 2005;49:1339-45.

20. Kneebone R. Evaluating clinical simulations for learning procedural skills: a theory-based approach. Acad Med 2005;80:549-53.

21. Kneebone R, Nestel D, Wetzel C, et al. The human face of simulation: patient-focused simulation training. Acad Med 2006;81: 919-24.

22. Weller J, Dowell A, Kljakovic M, et al. Simulation training for medical emergencies in general practice. Med Educ 2005;39: 1154.

23. Ravert P. An integrative review of computer-based simulation in the education process. Comput Inform Nurs 2002;20:203-8

24. Bowles LT. Recommendations for emergency medicine. Ann Emerg Med 1995;25:234-5.

25. Celenza A. Evolution of emergency medicine teaching for medical students. Emerg Med Australas 2006;18:219-20.

26. Coates WC, Gill AM, Jordan R. Emergency medicine clerkship directors: defining the characteristics of the workforce. Ann Emerg Med 2005;45:262-8.

27. Manthey DE, Coates WC, Ander DS, et al. Report of the task force on national fourth year medical student emergency medicine curriculum guide. Ann Emerg Med 2006;47:e1-7.

28. Ten Eyck RP, Maclean TA. Improving the quality of emergency medicine rotation/clerkship evaluations. Am J Emerg Med 1994;12:113-7.

Correspondence to: Dr. Trevor S. Langhan, Rm. C231-1403 29th St. NW, Calgary AB T2N 2T9; trevorlanghan@shaw.ca 